\title{
Socio-demographic and clinical correlates of parenting style among parents having ADHD children: a cross-section study
}

\author{
Ghada Mohamed Salah El-Deen ${ }^{*}$, Amira Mohamed Yousef, Amany Elshabrawy Mohamed and \\ Abdallah Saad Ibrahim
}

\begin{abstract}
Background: ADHD affects $7.8 \%$ of the school-aged population, making it one of the most common childhood brain illnesses. It is characterized by abnormally high levels of inattention, activity, and impulsivity at a young age. Being a parent of a child with ADHD is a real challenge, as the parents tend to be more disapproving, critical, and provide more impulse control directions; such parenting style can have an impact on the illnesses course, accentuate its signs and symptoms, and lead to secondary development of co-morbid psychiatric and behavioral problems. This makes the parent-child effect a matter of clinical importance that needs to be carefully assessed and managed. We aimed to estimate the sociodemographic and clinical correlates of parenting attitudes among parents having ADHD children. This cross-sectional study included 48 ADHD children from both sexes, aged from 6 to 12 years old, and their parents. In our study, we applied the Stanford-Binet Intelligence Scale 5th edition, the Conner's Parent Rating Scalerevised, the parenting style as perceived by children questionnaire, and the Fahmy and El-Sherbini questionnaire for the measurement of socioeconomic status.
\end{abstract}

Results: Mothers of ADHD children had significantly lower scores of over-protections parenting style than the fathers; the current study showed a significant increase in total parenting scores and warmth/support in mild ADHD cases than in moderate and severe ones, and there is a significant increase in the mother's positive parenting style toward ADHD children with lower levels of social problems, mild cases, and older age. There is a significant increase of positive parenting style toward ADHD children exerted by post graduated, professionally working, and high social class fathers and by working mothers among rural residents and high social class mothers. There is a positive correlation between IQ and a mother's warmth/support.

Conclusion: ADHD children with mild symptoms, higher social functioning of the child, high socioeconomic level of the family, better education, and professional occupations of parents were associated with positive parenting style.

Keywords: ADHD, Children, Parenting style

\section{Background}

Attention deficit hyperactivity disorder (ADHD) is a neurodevelopmental illness that affects children and is marked by hyperactivity, impulsivity, and inattention

*Correspondence: egyptrose2@gmail.com

Departement of psychiatry, Faculty of Medicine, Zagazig University, Zagazig, Egypt that are out of character for their age. According to DSM 5 , there are 3 subtypes of the disorder: inattentive type, hyperactive/impulsive type, and combined type [1].

ADHD has an early onset with a worldwide prevalence between 5 and 7.2\% [2]. It is diagnosed in boys about three times higher than in girls [3]. Prognosis, comorbidity, and persistence of ADHD symptoms through adolescence and adulthood are affected by many factors including family characteristics [4]. 
Parenting behavior is mainly composed of two interrelated dimensions; effective behavioral control, motional responsiveness to the child [5]. According to these two dimensions, there are four general broad styles of parenting: (1) authoritative parents, (2) authoritarian parents, (3) indulgent parents, (4) and neglectful parents [6].

Furthermore, certain parental attitudes were reported through research to be associated with an increase in children's behavioral and emotional problems such as discrimination, inconsistent discipline, overprotection, and harsh discipline [7]

Parents of ADHD children need to exert more effort to ameliorate the behavior, to support academic work, and to compensate their children deregulation. These parents tend to see the behavior of their children with mean intent [8] which increases conflict and stress. Some parents of children with ADHD especially mothers tend to work fewer hours or even quit their jobs because their children need more assistance [9]. Due to children's ADHD symptomology, their parents are less engaging and less warm than other parents of normal children [10]; they may respond with high levels of verbal aggression and disciplinary measures to troublesome behaviors [11], which makes their children respond negatively, influencing a bidirectional process. This process may negatively affect broad child outcomes, increase noncompliance in classroom and playground activities, and stealing among children with ADHD [12].

Demanding, temperamental, and uncooperative ADHD children have been noted to make it difficult for their parents to manage their behavior [13]. Temperament traits can be considered as an early emerging sign of behavioral tendencies that may create vulnerability for ADHD. Deater-Deckard et al. (2014) suggested that the family factors, such as parenting, influence the development and maintenance of self-regulation [14]. Negative parenting may thus interfere with the development of self-control [15], contributing to the rise and maintenance of child psychopathology, such as ADHD [16]. Also, children who have low control over temperament and are highly impulsive are more vulnerable to the negative consequences of bad parenting $[17,18]$.

Deault et al. (2010) found that ADHD is associated with problematic family functioning, including higher rates of parental psychopathology and conflicted parentchild relationships, exacerbated in children with comorbid ODD and CD [19].

Unlike the wide use of medication in the treatment of ADHD, psychosocial treatment is rare in most communities [20]. Psychosocial treatment needs to be evident to produce significant improvements in child functioning and health for communities to commit resources for it. A variety of behavioral parent training (BPT) protocols for parents of children with ADHD and associated behavior problems have been developed, BPT is an evidence-based psychosocial treatment that focuses on increasing positive and decreasing negative parenting through instruction and practice in effective caretaking strategies. It is intended to produce sustained improvement in child behavior indirectly through the promotion of effective parenting strategies. Parents are specifically instructed to engage in positive monitoring and attendance, set clear expectations, and provide consistent positive and negative outcomes [21].

\section{Method}

\section{Study site, design, and participant}

This study is cross-sectional. It was held at the Zagazig University Hospitals' out-patient child psychiatry clinic between November 1, 2020, and April 30, 2021.

Our study involved 48 ADHD children with their parents. A comprehensive sample was estimated to be 48 children fulfilling the diagnostic criteria of ADHD, collected during a period of 6 months (November 2020April 2021), as the rate of cases was approximately 8 cases/month. All the children who attended at the clinic for the first time during the period of sample collection with a complaint of inattentiveness and/or hyperactivity or who were newly diagnosed with ADHD during this period were screened to determine their eligibility for participation in the study according to specific inclusion and exclusion criteria. We included the children who have been diagnosed with ADHD according to the diagnostic criteria of DSM 5 and aged from 6 to 12 years old without chronic medical illness and their parents don't have another child with a chronic medical condition or psychiatric disorder. Both sex and all socioeconomic class were included. We excluded all the ADHD children who have parents with known chronic health problems or who have a history of psychiatric disorders. And parents who cannot read or write the Arabic language or those who refused to participate.

A written informed consent was obtained with the approval of the Ethical Committee.

\section{Study tools}

The following psychometric assessments were made to the participants enrolled in the study:

(a) Semi-structured interview for the child and one of or both parents according to the psychiatric sheet of child unite of Psychiatry Department of Zagazig University Hospital to collect detailed child psychiatric history and child evaluation through a comprehensive interview including mental status and physical examination. 
(b) The Arabic version Conner's Parent Rating Scalerevised $L$ [22]: It is a screening questionnaire prepared for the parents to detect if their children aged 3 to 17 have ADHD. It consists of 80 questions for parents to respond, each with four answers to choose one of them: 0 (not at all), 1 (just a little), 2 (pretty much), or 3 (very much). It assesses the severity of attention deficit, hyperactivity, and impulsive symptoms in addition to diagnosing ADHD. The National Institute of Mental Health approved the scale's validity, reliability, and stability. It was translated by Dr Al-Behairy A, AglaanA. (2009) [23].

(c) Stanford-Binet Intelligence Scale 5th edition: It was applied on the ADHD children. This test was designed to test the cognitive abilities and the intelligence; it is used for ages starting from 2 up to 89 years and provides a full-scale IQ. The SB5 index is composed of five components: fluid reasoning, knowledge, quantitative reasoning, visual-spatial processing, and working memory (subtests are grouped together to form one of the two domains or one of the five factor indices: The two domains or the five factor indexes are added together to obtain the full-scale IQ score [24]. Cronbach's alpha for the full-scale IQ scores were 0.97 to 0.98 . Verbal and nonverbal IQ scores have coefficients of 0.95 to 0.96 [25].

(d) Parenting style as perceived by children questionnaire: This scale includes 60 items divided into 5 subscales; one subscale represents positive parenting attitudes (warmth/support) and 4 subscales represent negative parenting attitudes: harsh control, inconsistency, overprotection, and discrimination. The subject replies to each phrase twice; the first is that represents his perception of the father's attitude while the other reflects the mother's attitude besidesthe total score. The items should be answered by the child, only by yes or no. All the items of the positive subscale are positive so that choosing yes as an answer for an item of this subscale equals 2 , while choosing no equals 1 and vice versa in the 4 negative subscales. The higher the score of the positive subscale the more warmth and support perceived by the child. On contrary, the higher the score of the negative subscales the less negative parental attitudes were perceived by the child. The total score ranges from 60 to 120 and the higher the score the more positive attitudes perceived by the child. This scale was developed in Arabic by Prof. Dr. Amany Abd El Maksoud, Menofia University, 2013 [26]

(e) Updated version of Fahmy and El-Sherbini Questionnaire for measurement of socioeconomic status
[27]: The updated scale had seven domains with a total score of 84 , with a higher score indicating higher socioeconomic status: occupation, education, culture, family possessions, home sanitation, economic, and health care. The updated scale included all the variables of the previous one. The updated scale was tested for content validity by two panels of the Psychiatric Department experts. These experts assessed the tools for clarity, relevance, comprehensiveness, applicability, and understanding.

The reliability of the updated scale was tested by measuring their internal consistency. It demonstrated an excellent level of reliability (Cronbach's alpha $=0.81$ ).

\section{Statistical analysis}

The collected data were computerized and statistically analyzed using the SPSS program (Statistical Package for Social Science) version 25.0 [28]. Qualitative data were represented as frequencies and relative percentages. Chisquare test was used to calculate the difference between qualitative variables. Quantitative data were expressed as mean $\pm \mathrm{SD}$ (Standard deviation). Independent $T$ test was used to calculate the difference between quantitative variables in two groups in normally distributed data. ANOVA $F$-test test was used to calculate the difference between quantitative variables in more than two groups in normally distributed data. Pearson correlation coefficient was used to calculate the correlation between quantitative variables.

\section{Results}

The current study found that the mean age of the studied group was 8.6 (6-12). Most of them were male (85.4\%), 1st child (58.3\%), in public school (56.2\%), and living in urban areas (54.2\%). The majority of their fathers' (47.9\%) and of their mothers' (60.4\%) educational level was secondary school or intermediate institute. Only $27.1 \%$ of their fathers had a professional job and most of their mothers $(64.6 \%)$ were housewives; most of their families (45.8\%) were of the middle social class. $29.2 \%$ of the parents of the studied group had positive consanguinity, and most of the studied children (68.7\%) had a family history of psychiatric problems: most frequent among parents (41.7\%).

As regard parenting scores among the studied group, this study found that mothers of ADHD children had significantly lower scores of overprotection parenting style (high levels of overprotection among mothers) than the fathers (Table 1).

In the relationship between child characteristics and parenting attitudes of the father, the current study 
Table 1 Parenting score among the studied group

\begin{tabular}{|c|c|c|c|c|}
\hline Parenting style & $\begin{array}{l}\text { Fathers } \\
(n=48)\end{array}$ & $\begin{array}{l}\text { Mothers } \\
(n=48)\end{array}$ & $T$ & $P$ \\
\hline \multicolumn{5}{|l|}{ Warmth/support } \\
\hline- Mean $\pm S D$ & $32.65 \pm 5.06$ & $34.38 \pm 4.30$ & 1.80 & 0.08 \\
\hline - Range & 20-39 & $24-40$ & & \\
\hline \multicolumn{5}{|l|}{ Overprotection } \\
\hline- Mean $\pm S D$ & $16.27 \pm 2.61$ & $14.04 \pm 2.74$ & 4.09 & $<0.001^{* *}$ \\
\hline - Range & $10-20$ & $10-20$ & & \\
\hline \multicolumn{5}{|l|}{ Harsh control } \\
\hline- Mean $\pm S D$ & $15.27 \pm 2.69$ & $14.94 \pm 2.76$ & 0.60 & 0.55 \\
\hline - Range & $10-20$ & $10-20$ & & \\
\hline \multicolumn{5}{|l|}{ Inconsistency } \\
\hline- Mean $\pm S D$ & $16.19 \pm 2.53$ & $15.83 \pm 2.81$ & 1.49 & 0.14 \\
\hline - Range & $10-20$ & $10-20$ & & \\
\hline \multicolumn{5}{|l|}{ Discrimination } \\
\hline- Mean $\pm S D$ & $17.9 \pm 2.67$ & $17.94 \pm 2.32$ & 0.08 & 0.94 \\
\hline - Range & $10-20$ & $10-20$ & & \\
\hline \multicolumn{5}{|l|}{ Total } \\
\hline- Mean $\pm S D$ & $98.02 \pm 10.82$ & $96.31 \pm 10.26$ & 0.79 & 0.43 \\
\hline - Range & $72-114$ & $78-112$ & & \\
\hline
\end{tabular}

**highly significant $(p<0.01)$

showed a significant increase in total parenting scores and warmth/support in mild ADHD cases than in moderate and severe (Table 2); as regard parenting attitudes of mothers, the study showed a significant increase in total and harsh control scores (means a low level of harsh control) among ADHD children aged from 10 to 12 years old, in total warmth/support and harsh control scores among ADHD children who have lower levels of social problems, and in total and warmth/support scores among mild cases (Table 3 ).

The relationship between family characteristics and parenting attitudes of fathers showed an increase in total warmth/support, harsh control, and inconsistency scores (means low levels of harsh control and inconsistency) among post-graduated educated fathers and increase in total warmth/support, harsh control, discrimination, and inconsistency scores (means low levels of harsh control, discrimination, and inconsistency) among professionally working fathers. There was an increase in warmth/support among high social class (Table 4), but the relationship with parenting attitudes of mothers showed an increase in total and inconsistency scores among post-graduated educated mothers, increase in total harsh control, discrimination, and inconsistency scores among professionally working mothers, increase in total score among rural residents, and an increase in total discrimination and inconsistency scores among high social class (Table 5).
Additionally, a positive correlation between IQ and mother's warmth/support among the studied group was found (Table 6).

\section{Discussion}

Parents of children with ADHD tend to use inappropriate parenting styles, they are more disapproving, critical, provide more impulse control directions and display poorer monitoring and more corporal punishment than parents of children without ADHD [29] trying to control disruptive behaviors which are of the main characteristics of the hyperactive-impulsive type of ADHD [30]. These parenting styles can affect the course of the illness, exacerbate its manifestations, and give rise to secondary development of co-morbid psychiatric and maladaptive behaviors such as oppositional defiant disorder (ODD) and aggression [31].

This study has therefore been carried out to evaluate the parenting attitudes of parents of those children and to explore their associations with child and family characteristics.

The results of this study revealed $48 \%$ of the studied group fathers and about $60 \%$ of the mother's educational level was a secondary school or intermediate institute, while about a third of the sample's fathers and less than a third of the sample's mothers were university graduates or post-graduates; these findings are consistent with [32]. A possible explanation of such a result could be that the poor parenting skills in low-educated parents may lead to negative attitudes in treating children which in turn could worsen the clinical presentation of ADHD which in turn push the family to seek help. However, this wasn't the case in other studies which found no significant association between parents' education and ADHD [33, 34].

Regarding the work of the parents, more than twothirds of the study populations were in non-professional occupations. This is consistent with [35] and in contrast, Al-Hamed et al. (2009) and Ford et al. (2004) found no association between father occupation and ADHD in their offspring $[36,37]$.

It was also noteworthy that about $65 \%$ of mothers were housewives. This finding is contrary to Malek et al. (2012) which have considered maternal employment as a probable predictive factor for ADHD [38]. In the present study, it seems that maternal employment may have played a protective role in regard to the mental health of the sample children due to better economic chances, higher education of employed mothers, and have more chances to interact with the external world and acquire a daily knowledge which may help mothers for better understanding of their children.

Regarding parenting attitudes, the current study shows a significant increase in maternal overprotection compared 
Table 2 Relationship between child characteristics and parenting attitudes of father

\begin{tabular}{|c|c|c|c|c|c|c|c|c|c|c|c|c|}
\hline \multicolumn{5}{|c|}{ Social problems } & \multicolumn{2}{|c|}{ Order of birth } & \multicolumn{2}{|c|}{ Age group } & \multicolumn{2}{|c|}{ Child Sex } & \multirow{2}{*}{\multicolumn{2}{|c|}{ 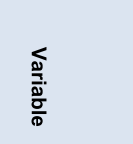 }} \\
\hline$\frac{\mathscr{O}}{\frac{0}{0}}$ & 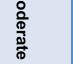 & $\underline{\underline{z}}$ & 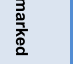 & 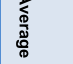 & $\overrightarrow{\underline{\omega}}$ & $\frac{\text { o }}{\frac{\sigma}{\omega}}$ & $\begin{array}{l}\overrightarrow{0} \\
\stackrel{\vec{N}}{ }\end{array}$ & $\dot{8}$ & $\frac{3}{\frac{3}{0}}$ & $\begin{array}{l}\frac{T}{\Phi} \\
\frac{\Phi}{\tilde{\omega}} \\
\frac{\omega}{\omega}\end{array}$ & & \\
\hline 103.88 .515 & 99.69 .55 & 98.779 .523 & 88.94 .74 & 89.19 .78 & $95.04 \pm 10.2$ & $98.1 \pm 10.32$ & $100.9 \pm 9.62$ & $94.21 \pm 9.98$ & $96.78 \pm 10.23$ & $93.57 \pm 10.85$ & $\begin{array}{c}\text { Mean } \pm \\
S D\end{array}$ & \\
\hline $85-111$ & 82-111 & $80-112$ & $85-96$ & $78-107$ & 78-111 & $80-112$ & $80-112$ & $78-111$ & $78-112$ & $80-108$ & Range & \\
\hline \multicolumn{5}{|c|}{$<4.847$} & \multicolumn{2}{|c|}{1.02} & \multicolumn{2}{|c|}{2.19} & \multicolumn{2}{|c|}{0.761} & Test & $\frac{0}{0} \frac{0}{0}$ \\
\hline \multicolumn{5}{|c|}{$<0.001^{m *}$} & \multicolumn{2}{|c|}{0.31} & \multicolumn{2}{|c|}{$0.03^{*}$} & \multicolumn{2}{|c|}{0.45} & $\mathbf{P}$ & \\
\hline 373.117 & 36.83 .25 & 34.693.945 & 30.42 .57 & 32.24 .803 & 34.044 .247 & 34.854 .44 & \begin{tabular}{|l|}
35.674 .1 \\
\end{tabular} & 33.794 .321 & 34.564 .272 & 33.294 .645 & $\begin{array}{c}\text { Mean } \pm \\
S D\end{array}$ & \\
\hline $30-40$ & 30-39 & $27-39$ & $28-36$ & $24-39$ & $27-40$ & $24-39$ & $27-40$ & $24-39$ & $24-39$ & $28-40$ & Range & 둥 \\
\hline \multicolumn{5}{|c|}{4.884} & \multicolumn{2}{|c|}{-0.64} & \multicolumn{2}{|c|}{1.42} & \multicolumn{2}{|c|}{0.721} & Test & 홍 \\
\hline \multicolumn{5}{|c|}{$<0.001^{* *}$} & \multicolumn{2}{|c|}{0.52} & \multicolumn{2}{|c|}{0.16} & \multicolumn{2}{|c|}{0.47} & $\mathbf{P}$ & 75 \\
\hline 14.53 .207 & 12.81 .98 & $14.622 .844 \mid$ & 15.13 .58 & \begin{tabular}{|l|l}
13.42 .011 \\
\end{tabular} & \begin{tabular}{|l|l|l|} 
& 13.79 \\
2.699
\end{tabular} & 14.42 .817 & 14.732 .685 & 13.732 .742 & 14.22 .61 & 13.143 .485 & $\begin{array}{c}\text { Mean } \pm \\
S D\end{array}$ & 오 \\
\hline $10-18$ & $10-16$ & $10-19$ & $10-20$ & $10-17$ & $10-20$ & 10-19 & $10-18$ & $10-20$ & $10-20$ & $10-18$ & Range & 훙 \\
\hline \multicolumn{5}{|c|}{1.149} & \multicolumn{2}{|c|}{0.76} & \multicolumn{2}{|c|}{1.19} & \multicolumn{2}{|c|}{0.939} & Test & $\stackrel{\vec{D}}{\stackrel{a}{a}}$ \\
\hline \multicolumn{5}{|c|}{0.35} & \multicolumn{2}{|c|}{0.45} & \multicolumn{2}{|c|}{0.24} & \multicolumn{2}{|c|}{0.35} & $\mathbf{P}$ & 훙 \\
\hline 16.632 .326 & $\begin{array}{c}15.82 .61 \\
6 \\
\end{array}$ & 15.382 .873 & 13.72 .63 & 132.055 & 14.432 .821 & 15.652 .581 & 16.132.85 & 14.39 2.585 & 14.952 .655 & $\mid 14.863 .579$ & $\begin{array}{c}\text { Mean } \pm \\
S D\end{array}$ & $\stackrel{I}{\Xi}$ \\
\hline 13-19 & 11-19 & $10-20$ & $10-18$ & $11-18$ & 10-19 & $11-20$ & $10-20$ & $10-19$ & $10-20$ & $10-19$ & Range & 5 \\
\hline \multicolumn{5}{|c|}{3.126} & & 1.53 & & .09 & 0.0 & 082 & Test & $\overline{7}$ \\
\hline & & $0.02^{*}$ & & & & 0.13 & & $04^{*}$ & & .94 & $\mathbf{P}$ & 0 \\
\hline 16.882 .167 & $\begin{array}{c}16.32 .21 \\
4\end{array}$ & 15.462 .367 & 13.12 .41 & $\mid$\begin{tabular}{|l}
14.73 .743 \\
\end{tabular} & 15.363 .291 & 15.42 .037 & 16.072 .404 & 15.062 .957 & 15.442 .802 & 153.055 & $\begin{array}{c}\text { Mean } \pm \\
S D\end{array}$ & $\bar{\Xi}$ \\
\hline $14-20$ & 13-19 & $11-19$ & $10-17$ & $10-22$ & $10-22$ & $11-18$ & $11-20$ & $10-22$ & $10-20$ & $11-20$ & Range & $<\underline{\underline{\underline{w}}}$ \\
\hline & & 2.328 & & & & 0.05 & & .15 & 0.3 & 378 & Test & $\overline{8}$ \\
\hline & & 0.07 & & & & 0.96 & & .26 & 0.7 & .71 & $\mathbf{P}$ & ลี \\
\hline 19.380 .916 & 182.494 & 18.54 1.713 & 16.92 .04 & 16.73 .093 & 17.862 .49 & 18.052 .114 & 18.871 .727 & 17.522 .451 & 18.052 .213 & 17.292 .984 & $\begin{array}{c}\text { Mean } \pm \\
S D\end{array}$ & 뭄 \\
\hline $18-20$ & $14-20$ & $14-20$ & 13-19 & $10-20$ & $10-20$ & $13-20$ & $14-20$ & $10-20$ & $10-20$ & $13-20$ & Range & $=\overline{3} \cdot$ \\
\hline & & 2.312 & & & & 0.28 & & 1.92 & 0.8 & 801 & Test & $\overline{\mathrm{g}}$ \\
\hline & & 0.07 & & & & 0.78 & & .06 & & 43 & $\mathbf{P}$ & के \\
\hline
\end{tabular}

\begin{tabular}{|c|c|c|c|c|c|c|c|}
\hline \multicolumn{3}{|c|}{ Severity } & \multicolumn{3}{|c|}{ Subtype } & \multirow{2}{*}{\multicolumn{2}{|c|}{ Variable }} \\
\hline \multirow{2}{*}{ 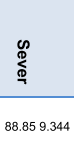 } & \multirow{2}{*}{$\begin{array}{l}\text { z. } \\
\frac{0}{0} \\
\frac{0}{2} \\
\frac{0}{\sigma} \\
98.188 .895\end{array}$} & \multirow{2}{*}{\begin{tabular}{|c|}
$\mathbf{z}$ \\
102.710 .468 \\
\end{tabular}} & \multirow{2}{*}{ 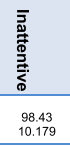 } & \multirow{2}{*}{ 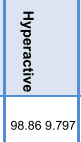 } & \multirow{2}{*}{ 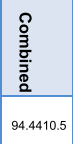 } & & \\
\hline & & & & & & $\begin{array}{c}\text { Mean } \pm \\
S D\end{array}$ & \multirow{2}{*}{ ๑ } \\
\hline 78-107 & $80-110$ & $85-112$ & $80-108$ & $82 \cdot 111$ & $78-112$ & Range & \\
\hline \multicolumn{3}{|c|}{6.496} & \multicolumn{3}{|c|}{1.028} & Test & \\
\hline \multicolumn{3}{|c|}{$<0.001^{* *}$} & \multicolumn{3}{|c|}{0.37} & $\mathbf{P}$ & \\
\hline 31.313 .8 & 35.363 .899 & 36.144 .259 & 363.916 & \begin{tabular}{|l|}
34.714 .358 \\
\end{tabular} & 33.784 .38 & $\begin{array}{c}\text { Mean } \pm \\
S D\end{array}$ & \\
\hline $24-38$ & $27-40$ & 30-39 & $28-40$ & $27-39$ & $24-39$ & Range & 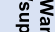 \\
\hline \multicolumn{3}{|c|}{5.518} & \multicolumn{3}{|c|}{0.797} & Test & 홍 \\
\hline \multicolumn{3}{|c|}{$0.01^{\star \star}$} & \multicolumn{3}{|c|}{0.46} & $\mathbf{P}$ & 75 \\
\hline 13.152 .4 & 14.292 .774 & 14.713 .094 & 13.292 .6 & \begin{tabular}{|l|}
$\mid 14.642 .763$ \\
\end{tabular} & 13.932 .77 & $\begin{array}{c}\text { Mean } \pm \\
S D\end{array}$ & ญ \\
\hline $10-17$ & $10-20$ & 10-18 & $10-17$ & 10-19 & $10-20$ & Range & $=\frac{7}{0}$ \\
\hline \multicolumn{3}{|c|}{1.007} & \multicolumn{3}{|c|}{0.619} & Test & $=$ \\
\hline \multicolumn{3}{|c|}{0.37} & \multicolumn{3}{|c|}{0.54} & $\mathbf{P}$ & के \\
\hline $\begin{array}{c}13.462 .0 \\
25\end{array}$ & 15.322 .816 & 16.142 .968 & $\begin{array}{c}15.573 .5 \\
05\end{array}$ & 15.57 2.277 & $\begin{array}{c}14.442 .79 \\
2 \\
\end{array}$ & $\begin{array}{c}\text { Mean } \pm \\
S D\end{array}$ & \\
\hline $11-18$ & 10-19 & $12-20$ & $10-18$ & $12-19$ & $10-20$ & Range & ฏ폻 \\
\hline \multicolumn{3}{|c|}{3.033} & \multicolumn{3}{|c|}{0.982} & Test & 흐의 \\
\hline \multicolumn{3}{|c|}{0.06} & \multicolumn{3}{|c|}{0.38} & $\mathbf{P}$ & \\
\hline $\begin{array}{c}14.623 .5 \\
95\end{array}$ & 15.322 .539 & 171.633 & $\begin{array}{c}15.433 .2 \\
59\end{array}$ & 161.922 & $\begin{array}{c}15.043 .10 \\
7\end{array}$ & $\begin{array}{c}\text { Mean } \pm \\
\text { SD }\end{array}$ & $\bar{\Xi}$ \\
\hline $10-22$ & $10-20$ & 14-19 & $11-20$ & 13-19 & $10-22$ & Range & 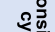 \\
\hline \multicolumn{3}{|c|}{1.699} & \multirow{2}{*}{\multicolumn{3}{|c|}{$\begin{array}{r}0.532 \\
0.59\end{array}$}} & Test & $\vec{\nabla}$ \\
\hline \multicolumn{3}{|c|}{0.19} & & & & $\mathbf{P}$ & 3 \\
\hline 172.708 & 18.182 .091 & 18.712 .215 & 18.142 .6 & $\begin{array}{l}18.57 \\
1.505 \\
\end{array}$ & 17.562 .57 & $\begin{array}{c}\text { Mean } \pm \\
S D\end{array}$ & $\frac{7}{n}$ \\
\hline $10-20$ & $13-20$ & $14-20$ & $14-20$ & $15-20$ & $10-20$ & Range & $\frac{n}{3}$ \\
\hline \multicolumn{3}{|c|}{1.65} & \multicolumn{3}{|c|}{0.913} & Test & 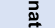 \\
\hline & 0.2 & & & 0.41 & & $\mathbf{P}$ & 훙 \\
\hline
\end{tabular}


Table 3 Relationship between child characteristics and parenting attitudes of mothers

\begin{tabular}{|c|c|c|c|c|c|c|c|c|c|c|c|c|}
\hline \multicolumn{5}{|c|}{ Social problems } & \multicolumn{2}{|c|}{ Order of birth } & \multicolumn{2}{|c|}{ Age group } & \multicolumn{2}{|c|}{ Child Sex } & \multirow{2}{*}{\multicolumn{2}{|c|}{ 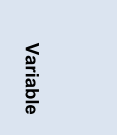 }} \\
\hline$\frac{\underline{\omega}}{\overline{\underline{\underline{E}}}}$ & $\begin{array}{l}3 \\
\frac{3}{0} \\
\frac{0}{9} \\
\frac{0}{0} \\
\frac{3}{0}\end{array}$ & $\underline{\underline{z}}$ & 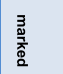 & 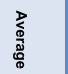 & $\vec{\mu}$ & $\frac{0}{\frac{5}{6}}$ & $\frac{\overrightarrow{0}}{\vec{N}}$ & : & $\frac{3}{\frac{3}{\sigma}}$ & $\begin{array}{l}\frac{\pi}{\pi} \\
\frac{\underline{\omega}}{\omega}\end{array}$ & & \\
\hline 103.88 .515 & 99.69 .55 & 98.779 .523 & 88.94 .74 & 89.19 .78 & $95.04 \pm 10.2$ & $98.1 \pm 10.32$ & $100.9 \pm 9.62$ & $94.21 \pm 9.98$ & $96.78 \pm 10.23$ & $93.57 \pm 10.85$ & $\begin{array}{c}\text { Mean } \pm \\
S D\end{array}$ & \\
\hline $85-111$ & $82-111$ & $80-112$ & $85-96$ & 78-107 & $78-111$ & $80-112$ & $80-112$ & $78-111$ & $78-112$ & $80-108$ & Range & $n-1$ \\
\hline \multicolumn{5}{|c|}{$<4.847$} & \multicolumn{2}{|c|}{1.02} & \multicolumn{2}{|c|}{2.19} & \multicolumn{2}{|c|}{0.761} & Test & 응 \\
\hline \multicolumn{5}{|c|}{$<0.001^{* *}$} & \multicolumn{2}{|c|}{0.31} & \multicolumn{2}{|c|}{$0.03^{*}$} & \multicolumn{2}{|c|}{0.45} & $\mathbf{P}$ & \\
\hline 373,117 & 36.83 .25 & 34.693 .945 & 30.42 .57 & 32.24 .803 & 34.044 .247 & 34.854 .44 & 35.674 .1 & 33.794 .321 & 34.56 4.272 & 33.294 .645 & $\begin{array}{c}\text { Mean } \pm \\
\text { SD }\end{array}$ & \\
\hline $30-40$ & $30-39$ & $27-39$ & $28-36$ & $24-39$ & $27-40$ & $24-39$ & $27-40$ & $24-39$ & $24-39$ & $28-40$ & Range & క్ \\
\hline \multicolumn{5}{|c|}{4.884} & \multirow{2}{*}{\multicolumn{2}{|c|}{-0.64}} & \multicolumn{2}{|c|}{1.42} & \multirow{2}{*}{\multicolumn{2}{|c|}{$\begin{array}{l}0.721 \\
0.47\end{array}$}} & Test & 응 \\
\hline \multicolumn{5}{|c|}{$<0.001 *$} & & & \multicolumn{2}{|c|}{0.16} & & & $\mathbf{P}$ & 75 \\
\hline 14.53 .207 & 12.81 .98 & 14.622 .844 & 15.13 .58 & 13.42 .011 & 13.792 .699 & 14.42 .817 & 14.732 .685 & 13.732 .742 & 14.22 .61 & 13.143 .485 & $\begin{array}{c}\text { Mean } \pm \\
S D\end{array}$ & ఖ \\
\hline 10-18 & 10-16 & $10-19$ & $10-20$ & $10-17$ & 10-20 & $10-19$ & 10-18 & $10-20$ & $10-20$ & 10-18 & Range & 궁 \\
\hline \multicolumn{5}{|c|}{1.149} & \multirow{2}{*}{\multicolumn{2}{|c|}{$\begin{array}{l}0.76 \\
0.45\end{array}$}} & \multicolumn{2}{|c|}{1.19} & \multicolumn{2}{|c|}{0.939} & Test & \\
\hline \multicolumn{5}{|c|}{0.35} & & & \multicolumn{2}{|c|}{0.24} & \multicolumn{2}{|c|}{0.35} & $\mathbf{P}$ & 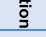 \\
\hline 16.632 .326 & $\begin{array}{c}15.82 .61 \\
6 \\
\end{array}$ & 15.382 .873 & \begin{tabular}{|l|l}
13.72 .63 \\
\end{tabular} & 132.055 & 14.432 .821 & \begin{tabular}{|l|}
15.652 .581 \\
\end{tabular} & 16.132 .85 & 14.392 .585 & 14.95 2.655 & $\mid$\begin{tabular}{|l|l|} 
& 14.863 .57 \\
\end{tabular} & $\begin{array}{c}\text { Mean } \pm \\
S D\end{array}$ & $\frac{\pi}{\underline{T}}$ \\
\hline 13-19 & 11-19 & $10-20$ & 10-18 & 11-18 & 10-19 & $11-20$ & 10-20 & $10-19$ & $10-20$ & 10-19 & Range & \\
\hline \multicolumn{5}{|c|}{3.126} & & 1.53 & 2.0 & 09 & & 082 & Test & $\bar{F}$ \\
\hline & & $0.02^{*}$ & & & & 0.13 & 0.0 & $84^{*}$ & 0.5 & 94 & $\mathbf{P}$ & o \\
\hline 16.882 .167 & $\begin{array}{c}16.32 .21 \\
4\end{array}$ & 15.462 .367 & $\begin{array}{l}13.12 .41 \\
\end{array}$ & 14.73 .743 & $\mid$\begin{tabular}{|l|l|} 
& 15.36 \\
3.2291
\end{tabular} & 15.42 .037 & 16.072 .404 & 15.062 .957 & \begin{tabular}{|l|l|} 
& 15.442 .802 \\
\end{tabular} & \begin{tabular}{|l|}
153.055 \\
\end{tabular} & $\begin{array}{c}\text { Mean } \pm \\
\text { SD }\end{array}$ & $\overline{\bar{z}}$ \\
\hline $14-20$ & 13-19 & $11-19$ & $10-17$ & $10-22$ & $10-22$ & $11-18$ & $11-20$ & $10-22$ & $10-20$ & $11-20$ & Range & $<\frac{\underline{\underline{v}}}{\underline{\underline{w}}}$. \\
\hline & & 2.328 & & & & 0.05 & 1.1 & 15 & & 378 & Test & $\frac{\bar{m}}{\sigma}$ \\
\hline & & 0.07 & & & & 0.96 & 0.2 & 26 & 0.7 & 71 & $\mathbf{P}$ & ڤै \\
\hline 19.380 .916 & 182.494 & 18.541 .713 & 16.92 .04 & 16.73 .093 & 17.862 .49 & 18.052 .114 & 18.871 .727 & 17.522 .451 & 18.052 .213 & 17.292 .984 & $\begin{array}{c}\text { Mean } \pm \\
S D\end{array}$ & $\frac{D}{n}$ \\
\hline $18-20$ & $14-20$ & $14-20$ & 13-19 & $10-20$ & $10-20$ & $13-20$ & $14-20$ & $10-20$ & $10-20$ & $13-20$ & Range & $=$ = \\
\hline & & 2.312 & & & & 0.28 & -1.5 & 92 & & 801 & Test & 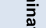 \\
\hline & & 0.07 & & & & 0.78 & 0.0 & 06 & 0.4 & 43 & $\mathbf{P}$ & t \\
\hline
\end{tabular}

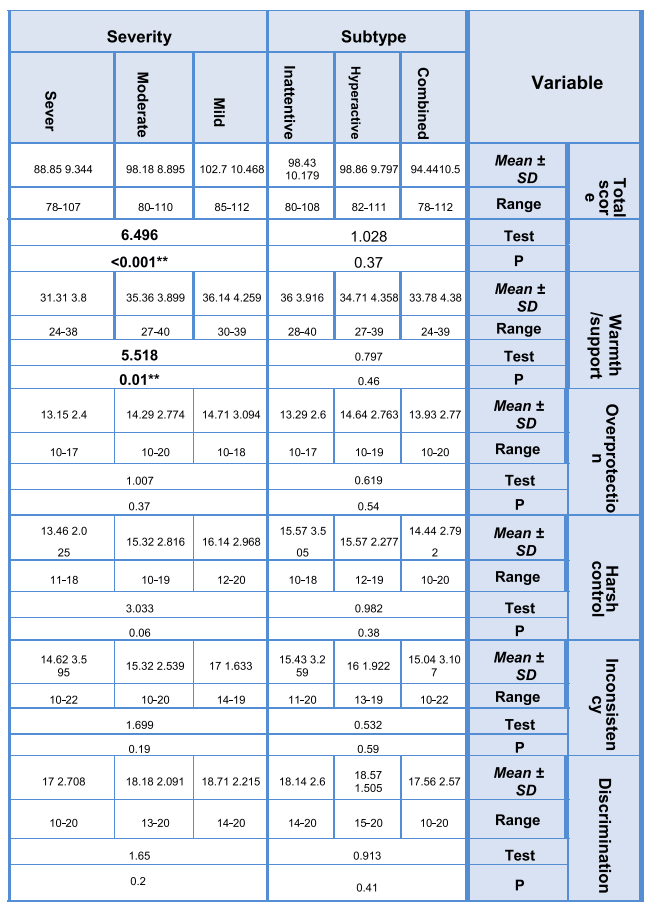

$S d$ standard deviation, $t$ independent $t$ test, $F$ ANOVA test, $N S$ non-significant $(P>0.05)$

*Significant $(P<0.05)$

to fathers. In reviewing the literature, prior studies have documented this association between maternal overprotection and ADHD symptoms of their children $[39,40]$. On the contrary, Khafi et al. (2019) found no association [41].
The current study shows that children whose ages ranging from 10 to 12 perceived their mothers' attitudes as more positive and less harsh and controlling than younger children. This result could be explained by the 


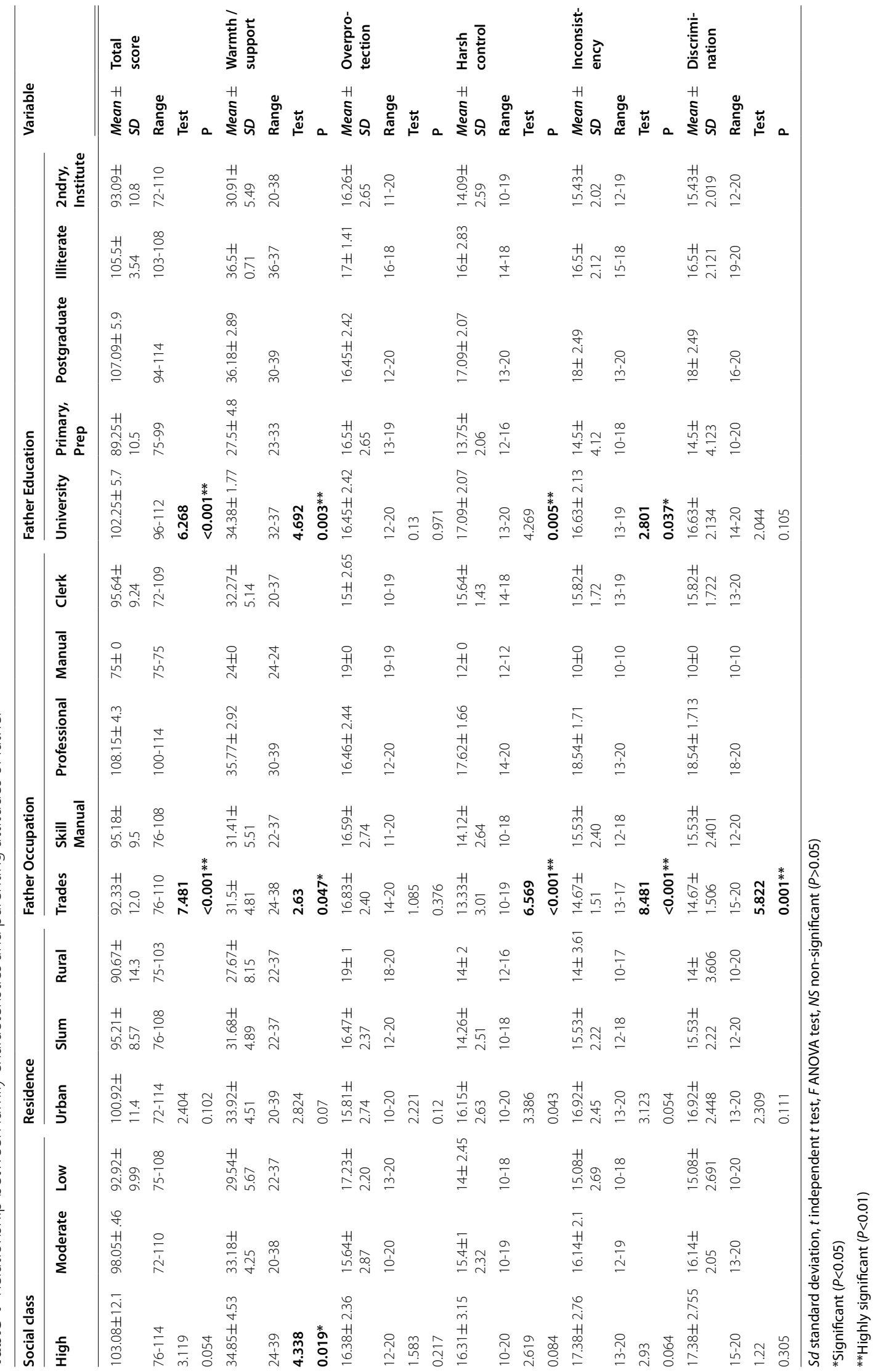




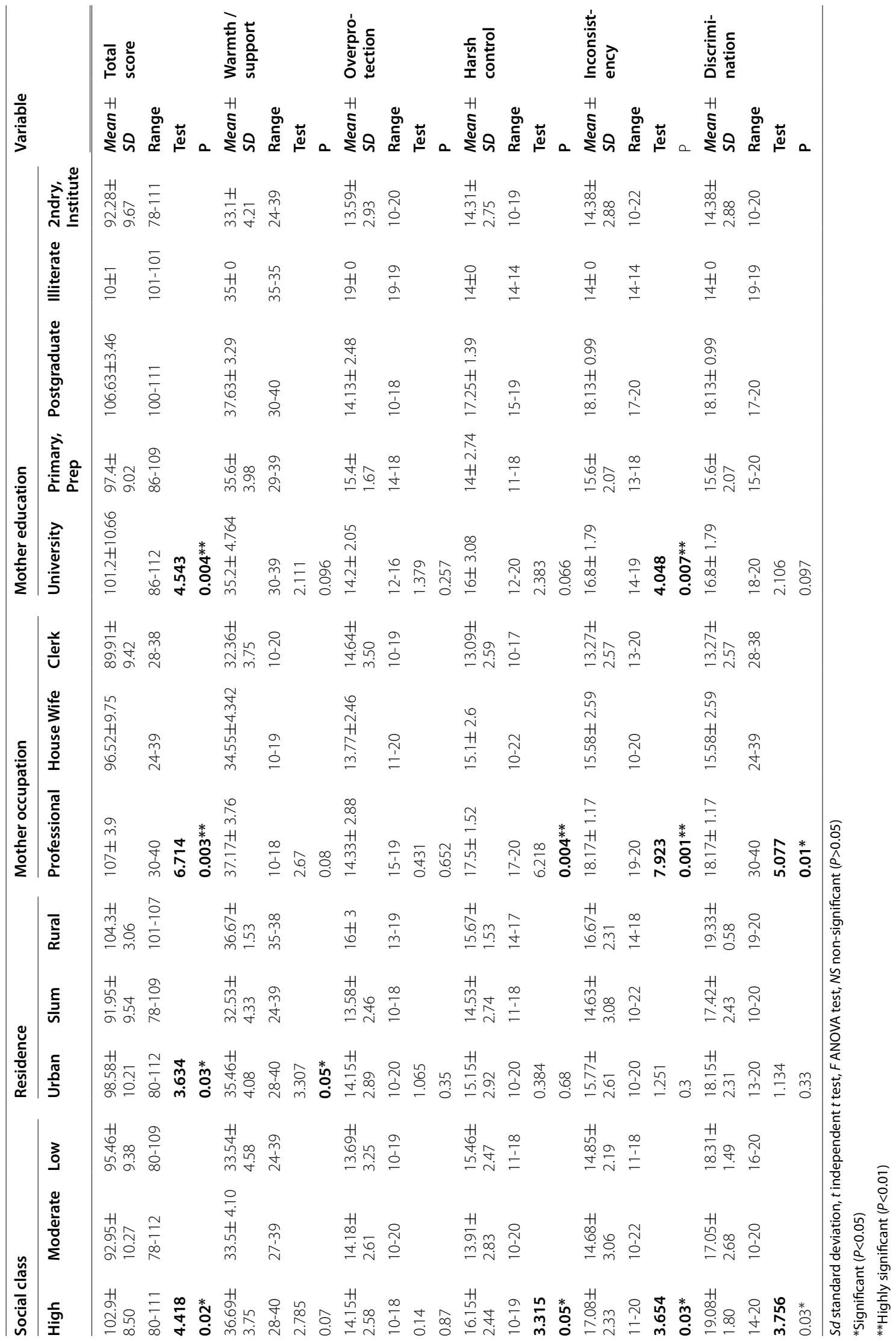


Table 6 Correlation between $\mathrm{IQ}$ and parenting among the studied group

\begin{tabular}{llll}
\hline Variable & & \multicolumn{2}{l}{$\begin{array}{l}\text { IQ score } \\
(\boldsymbol{n}=\mathbf{4 8})\end{array}$} \\
\cline { 3 - 4 } & & $\boldsymbol{r}$ & $\boldsymbol{P}$ \\
\hline Father & - Total & 0.22 & $0.13 \mathrm{NS}$ \\
& - Warmth/ support & 0.24 & $0.11 \mathrm{NS}$ \\
& - Overprotection & 0.14 & $0.35 \mathrm{NS}$ \\
& - Harsh control & 0.25 & $0.09 \mathrm{NS}$ \\
& - Inconsistency & 0.09 & $0.53 \mathrm{NS}$ \\
Mother & - Discrimination & 0.14 & $0.34 \mathrm{NS}$ \\
& - Total & 0.28 & $0.05 \mathrm{NS}$ \\
& - Warmth/ support & 0.42 & $\mathbf{0 . 0 0 4}$ ** \\
& - Overprotection & 0.05 & $0.71 \mathrm{NS}$ \\
& - Harsh control & 0.24 & $0.11 \mathrm{NS}$ \\
& - Inconsistency & 0.15 & $0.32 \mathrm{NS}$ \\
& - Discrimination & 0.06 & $0.67 \mathrm{NS}$ \\
\hline
\end{tabular}

$r$ Pearson's correlation coefficient, $N S$ non-significant $(P>0.05)$

*Significant $(P<0.05)$

**highly significant $(p<0.01)$

notion of a delayed developmental trajectory in ADHD rather than a fixed deficit [42]. Since older children are more capable of controlling their disruptive behavior, this could positively reflect on their relationship with their parents and more obviously with their mothers as they are more likely to spend longer time with them than fathers.

If we now turn to the relation between parenting attitudes and severity of ADHD, the results of this study show that children with mild ADHD reported their parents' attitudes as more positive and warmer. This finding is consistent with Choenni et al. (2019) who found that higher levels of maternal sensitivity were associated with low severe forms of ADHD [43]. Additionally, Nelson et al. (2019) discovered that extreme child hyperactivity at the age of 5.5 years enhanced mothers' anger toward their children at the age of 10, leading to higher delinquency and aggressiveness in adolescence [44]. On the contrary, Johnston et al. (2002) found that maternal responsiveness was not related to the severity of the child's ADHD symptoms but the child's conduct problems [45].

Regarding the relation between parenting attitudes and social functioning, the current results show an association between children with average social problems and more positive, warm, supportive, and less harsh mothers' attitudes. These findings are consistent with those of Bhide et al. (2019) who discovered that greater parenting warmth and lower parenting anger were related to increased child pro-social behavior, self-control, and responsibility among children with ADHD [46]. Furthermore, Fenesy et al. (2019) discovered that child ADHD symptoms and poor parenting interactions were the only factors that predicted independent parent- and teacherrated social issues [47].

Regarding the relation between parenting attitudes and family characteristics, a significant result of the current study was the association between families of high social class and more positive, consistent, and less discriminating parenting attitudes of mother and more warm and supportive parenting attitudes of the father. These findings are in line with Russell et al. (2015) who observed that fathers with higher salaries reported greater participation with their children and that financial troubles in those families have adverse effects on aspects of parenting and the family/home environment [48].

In regard to parenting attitudes concerning parents' characteristics, results show that there were associations between more positive, consistent, and less harsh parenting attitudes with post-graduated parents and parents with professional professions. In reviewing the literature, we found a small body of research regarding this domain. However, one study by Johnston et al. (2002) found that better-educated mothers were more responsive with their children diagnosed with ADHD, and responsiveness was significantly, negatively related to reports of harsh parenting strategies [45]. If it is possible to hypothesize that parenting stress is negatively related to positive parenting, thus the current results are not in accordance to Anastopoulos et al. (1992), Harrison and Sofronoff (2002), and Mash and Johnston (1983) who discovered that maternal education, financial difficulties, and socioeconomic status are unrelated to parenting stress in families with ADHD children [49-51]. Our finding could be explained by the fact that higher educational level and professional occupations of parents could increase family income; decrease stress related to finance and in turn could provide children with better medical and psychological care that would improve their symptoms. Moreover, high education could help parents to better understand the nature of ADHD and in turn better handle their children's behavior. All of these factors could improve the parent-child relationship.

Another significant result in regard to family characteristics was the association between rural residents and more positive parenting attitudes of mothers than children from other areas. Due to the small number of children from rural areas in the current study, this result should be regarded with caution. The current results could be because living in urban areas could impose more stress on parents than living in rural in terms of social support, safe environment, 
and living expenses which could be easier to attain in rural areas than in urban areas. No data on this relation with ADHD was discovered in a study of the literature. Studies on parenting of normally developing children, on the other hand, were discovered. But Wahini and Ismawati (2005) found that urban moms experience more violence and neglect than rural moms [52]; Dewanggi et al. (2015) revealed that maternal acceptance was higher in urban than rural areas, while abuse, neglect, and maternal rejection were greater in rural regions [53].

We found a positive correlation between a child's IQ and mother warmth and support, and this finding supports the finding of Craig et al. (2019) who observed a negative relationship between total parenting stress of mothers and IQ of children with ADHD [54] and the finding of Khafi et al. (2019) who observed that IQ of the child with ADHD was negatively related to maternal insensitivity in treating her child [41].

Finally, our findings cannot be interpreted apart from few study limitations: first, the relatively small sample size; second, our sample did not include a control group which could add more value to our results; third, the potential biases introduced by the use of retrospective and self-report data collection must be assumed. Some information was drawn from history such as a psychiatric history of the family. Fourth, because this was a cross-sectional study, the causal factors or directivity of the associations could not be determined; therefore, future longitudinal studies are required.

\section{Conclusions}

ADHD children with mild symptoms, higher social functioning of the child, high socioeconomic level of the family, better education, and professional occupations of parents were associated with positive parenting.

\footnotetext{
Abbreviations

ADHD: Attention deficit hyperactivity disorder; DSM 5: Diagnostic and Statistical Manual of Mental Disorders-5; BPT: Behavioral parent training; SPSS: Statistical Package for Social Science; SD: Standard deviation; ODD: Oppositional defiant disorder; IQ: Intelligence quotient.
}

\section{Acknowledgements}

Not applicable

\section{Authors' contributions}

GMS, AMY, AEM, and ASI designed the work and applied the psychometric assessment. GMS, AMY, AEM, and ASI interviewed the participants for diagnosing the psychiatric disorders if present. All authors contributed to the conception, preparation, and writing of this article. AMY gave the paper a final review and GMS forwarded the manuscript for publication. All authors have read and approved the manuscript.

\section{Funding}

The authors declare that they have no funding support.

\section{Availability of data and materials}

Not applicable

\section{Declarations}

\section{Ethics approval and consent to participate}

Official permissions were obtained from Institutional Review Board at the Faculty of Medicine, Zagazig University hospitals with reference number (ZUIRB\#4721) on 25-6-2020, and the Psychiatry Department same University. A written informed consent was obtained from all participants, and written informed consent was obtained from a parent or guardian for participants under 16 years old. and they had the right to withdraw from the study at any time and without negatively affecting their medical care. The results of this study could be used as a scientific publication, but the participant's identity will be confidential.

\section{Consent for publication \\ Not applicable}

\section{Competing interests}

The authors declare that they have no competing interests.

Received: 5 July 2021 Accepted: 9 October 2021

Published online: 02 December 2021

\section{References}

1. American Psychiatric Association (2013) The Diagnostic and Statistical Manual of Mental Disorders 5th Edition: DSM-5. American Psychiatric Association Press, Washington, DC

2. Thomas R, Sanders S, Doust J, Beller E, Glasziou P (2015) Prevalence of attention-deficit/hyperactivity disorder: a systematic review and metaanalysis. Pediatrics 135(4):e994-e1001

3. Daley D, Van der Oord S, Ferrin M, Danckaerts M, Doepfner M, Cortese S, Sonuga-Barke EJS, European ADHD Guidelines Group (2014) Behavioral interventions in attention-deficit/hyperactivity disorder: a meta-analysis of randomized controlled trials across multiple outcome domains. J Am Acad Child Adolesc Psychiatry 53(8):835-847

4. Tarver J, Daley D, Sayal K (2015) Beyond symptom control for attentiondeficit hyperactivity disorder (ADHD): what can parents do to improve outcomes? Child Care Health Dev 41(1):1-14

5. Rothbaum F, Weisz JR (1994) Parental caregiving and child externalizing behavior in nonclinical samples: A meta-analysis. Psychol Bull 116(1):55

6. Linver MR, Brooks-Gunn J, Kohen DE (2002) Family processes as pathways from income to young children's development. Dev Psychol 38(5):719-723

7. Ryan R, O'Farrelly C, Ramchandani P (2017) Parenting and child mental health. London J Prim Care (Abingdon) Nov 9(6):86-94

8. Johnston C, Seipp C, Hommersen P, Hoza B, Fine S (2005) Treatment choices and experiences in attention deficit and hyperactivity disorder: relations to parents' beliefs and attributions. Child Care Health Dev 31(6):669-677

9. Sharif F, Zarei S, Shooshtari AA, Vossoughi M (2015) The effect of stress management program using cognitive behavior approach on mental health of the mothers of the children with attention deficit hyperactivity disorder. Iran J Pediatr 25(3):1-11

10. Tripp G, Schaughency EA, Langlands R, Mouat K (2007) Family interactions in children with and without ADHD. J Child Fam Stud 16(3):385-400

11. Modesto-Lowe V, Danforth JS, Brooks D (2008) ADHD: does parenting style matter? Clin Pediatr 47(9):865-872

12. Anderson CA, Hinshaw SP, Simmel C (1994) Mother-child interactions in ADHD and comparison boys: Relationships with overt and covert externalizing behavior. J Abnorm Child Psychol 22(2):247-265

13. Johnston C, Mash EJ (2001) Families of children with attention-deficit/ hyperactivity disorder: review and recommendations for future research. Clin Child Fam Psychol Rev 4(3):183-207

14. Deater-Deckard K (2014) Family matters: Intergenerational and interpersonal processes of executive function and attentive behavior. Curr Dir Psychol Sci 23(3):230-236

15. Bernier A, Carlson SM, Deschênes M, Matte-Gagné C (2012) Social factors in the development of early executive functioning: A closer look at the caregiving environment. Dev Sci 15(1):12-24 
16. Kim J, Deater-Deckard K (2011) Dynamic changes in anger, externalizing and internalizing problems: Attention and regulation. J Child Psychol Psychiatry 52(2):156-166

17. Kiff CJ, Lengua LJ, Zalewski M (2011) Nature and nurturing: Parenting in the context of child temperament. Clin Child Fam Psychol Rev 14(3):251-260

18. Ullsperger JM, Nigg JT, Nikolas MA (2016) Does Child Temperament Play a Role in the Association Between Parenting Practices and Child Attention Deficit/Hyperactivity Disorder? J Abnorm Child Psychol 44(1):167-178

19. Deault LC (2010) A systematic review of parenting in relation to the development of comorbidities and functional impairments in children with attention-deficit/hyperactivity disorder (ADHD). Child Psychiatry Hum Dev 41(2):168-192

20. Zima BT, Bussing R, Tang L, Zhang L, Ettner S, Belin TR, Wells KB (2010) Quality of care for childhood attention-deficit/hyperactivity disorder in a managed care Medicaid program. J Am Acad Child Adolesc Psychiatry 49(12):1225-1237

21. Haack LM, Villodas MT, McBurnett K, Hinshaw S, Pfiffner LJ (2016) Parenting mediates symptoms and impairment in children with ADHDinattentive type. J Clin Child Adolesc Psychol 45(2):155-166

22. Conners, C. K. (2001): CRS-R: Conners'Rating Scales-revised: Technical Manual. Multi-Health Systems.

23. Al-Behairy A, Aglaan A (2009) Conner's' Rating scales. Dar El Nahda, Egypt

24. Roid GH, Pomplun M (2012) The Stanford-Binet Intelligence Scales. The Guilford Press

25. Pomplun M, Custer M (2005) The construct validity of the StanfordBinet 5 measures of working memory. Assessment 12:338-346

26. El-Maksoud (2011) Parenting styles scale, Dar Farha for distribution and publishing

27. Fahmy S, El-Sherbini AF (1983) Determining simple parameters for social classifications for health research. Bull High Institute Public Health 13(5):95-108

28. IBMCorp Ibm, S. P. S. S. (2017) statistics for windows, version 25.0. IBM Corp., Armonk, NY

29. Wirth A, Reinelt T, Gawrilow C, Schwenck C, Freitag CM, Rauch WA (2019) Examining the relationship between children's ADHD symptomatology and inadequate parenting: The role of household chaos. J Atten Disord 23(5):451-462

30. Robin AL (2014) Family therapy for adolescents with ADHD. Child and Adolescent Psychiatric Clinics 23(4):747-756

31. Tung I, Brammer WA, Li JJ, Lee SS (2015) Parenting behavior mediates the intergenerational association of parent and child offspring ADHD symptoms. J Clin Child Adolescent Psychol 44(5):787-799

32. Farid MN, Sabbour SM, Osman MH (2008) Prevalence and risk factors of Attention Deficit Hyperactivity among school children. Egypt J Commun Med 26(4):13-23

33. El-Nemr MF, Badr SH, Salem SM (2015) Prevalence of Attention Deficit Hyperactivity Disorder in Children. Sci J Public Health 2(3):274-280

34. Al Hamed JH, Taha AZ, Sabra AA, Bella H (2008) Attention deficit hyperactivity disorder (ADHD): Is it a Health Problem among Male Primary School Children. J Egypt Public Health Assoc 83(5):165-182

35. Kotimaa AJ, Moilanen I, Taanila A, Ebeling H, Smalley SL, Mcgough JJ, Järvelin MR (2003) Maternal smoking and hyperactivity in 8-year-old children. J Am Acad Child Adolesc Psychiatry 42(7):826-833

36. Al Hamed JH, Taha AZ, Sabra AA, Bella H (2009) Attention deficit hyperactivity disorder (ADHD): is it a health problem among male primary school children. Bahrain Med Bull 30(2):67-71

37. Ford T, Goodman R, Meltzer H (2004) The relative importance of child, family, school and neighbourhood correlates of childhood psychiatric disorder. Soc Psychiatry Psychiatr Epidemiol 39(6):487-496

38. Malek A, Amiri S, Sadegfard M, Abdi S, Amini S (2012) Associated factors with attention deficit hyperactivity disorder (ADHD): a case-control study. Arch Iran Med 15(9):560-563

39. Gar NS, Hudson JL (2008) An examination of the interactions between mothers and children with anxiety disorders. Behav Res Ther 46(12):1266-1274

40. Raishevich N, Kennedy SJ, Rapee RM (2010) Expressed emotion displayed by the mothers of inhibited and uninhibited preschool-aged children. J Clin Child Adolesc Psychol 39(2):187-194
41. Khafi TY, Borelli JL, Yates TM (2019) Prospective Associations between Maternal Self-Sacrifice/Overprotection and Child Adjustment: Mediation by Insensitive Parenting. J Child Fam Stud 28(1):202-217

42. Shaw P, Gilliam M, Liverpool M, Weddle C, Malek M, Sharp W et al (2011) Cortical development in typically developing children with symptoms of hyperactivity and impulsivity: support for a dimensional view of attention deficit hyperactivity disorder. Am J Psychiatr 168(2):143-151

43. Choenni V, Lambregtse-van den Berg MP, Verhulst FC, Tiemeier H, Kok R (2019) The longitudinal relation between observed maternal parenting in the preschool period and the occurrence of child ADHD symptoms in middle childhood. J Abnorm Child Psychol 47(5):755-764

44. Nelson T, East P, Delva J, Lozoff B, Gahagan S (2019) Children's inattention and hyperactivity, mother's parenting, and risk behaviors in adolescence: a 10-year longitudinal study of Chilean children. J Dev Behav Pediatr 40(4):249-256

45. Johnston C, Murray C, Hinshaw SP, Pelham WE Jr, Hoza B (2002) Responsiveness in interactions of mothers and sons with ADHD: relations to maternal and child characteristics. J Abnorm Child Psychol 30(1):77-88

46. Bhide S, Sciberras E, Anderson V, Hazell P, Nicholson JM (2019) Association between parenting style and socio-emotional and academic functioning in children with and without ADHD: a community-based study. J Atten Disord 23(5):463-474

47. Fenesy MC, Teh SE, Lee SS (2019) Negative Parenting Moderates the Prospective Association of ADHD Symptoms and Youth Social Problems. J Abnorm Child Psychol 40(3):1-15

48. Russell AE, Ford T, Russell G (2015) Socioeconomic associations with ADHD: findings from a mediation analysis. PLoS One 10(6):436-445

49. Anastopoulos AD, Guevremont DC, Shelton TL, DuPaul GJ (1992) Parenting stress among families of children with attention deficit hyperactivity disorder. J Abnorm Child Psychol 20(4):503-520

50. Harrison C, Sofronoff K (2002) ADHD and parental psychological distress: Role of demographics, child behav-ioral characteristics, and parental cognitions. J Am Acad Child Adolesc Psychiatry 41(3):703-711

51. Mash EJ, Johnston C (1983) Parental perceptions of child behavior problems, parenting self-esteem, and mothers' reported stress in younger and older hyperactive and normal children. J Consult Clin Psychol 51(1):86-99

52. Wahini M, Ismawati R (2005) Pengaruh pola asuh penerimaan-penolakan dan karakteristik anak terhadap perilaku anak diperkotaan dan perdesaan. Lentera Jurnal Studi Perempuan 1(2):119-127

53. Dewanggi M, Hastuti D, Herawati T (2015) The influence of attachment and quality of parenting and parenting environment on children's character in rural and urban areas of Bogor. Jurnal Ilmu Keluarga \& Konsumen 8(1):20-27

54. Craig F, De Giacomo A, Operto FF, Margari M, Trabacca A, Margari L (2019) Association between feeding/mealtime behavior problems and internalizing/externalizing problems in autism spectrum disorder (ASD), other neurodevelopmental disorders (NDDs) and typically developing children. Minerva Pediatr. https://doi.org/10.23736/S00264946.19.05371-4

\section{Publisher's Note}

Springer Nature remains neutral with regard to jurisdictional claims in published maps and institutional affiliations. 